\title{
Highlights from Recent Literature
}

\section{ANALYTICAL}

\subsection{Ultimate Precision in Flame Atomic Absorption Sprectrophotometry (FAAS) and its Application in the Determination of Gold}

L. and K. Li (Guangpu Shiyanshi, 1997, 14(6), 65-

67) of the Yunnan Province Analytical \& Testing Research Institute, Kunming, P.R. China have developed a very precise method of analysis for gold using flame atomic absorption spectrophotometry (FAAS). Two standards are prepared: one has slightly below and another has slightly above the sample absorbance, and the difference between the standards is small. The method was applied to the determination of gold and was found to have a good accuracy and precision. The relative standard deviation was only $0.2 \%-0.1 \%$ for microgram per litre concentrations of gold; and the method is simple and practical.

\subsection{Determination of the Purity of Gold Alloys using $\gamma$-ray Transmission Techniques}

Work at the Toklo Metropolitan Industrial Technology Research Institute, Tokyo, by T. Suzuki, R. Kitsutaka, T. Muto and S. Morisaki (Jpn. J. Appl. Phys, Part 1, 1998, 37, 6242-6247) has demonstrated the use of triple and dual energy $\gamma$-ray transmission (TEGAT and DEGAT) techniques to determine non-destructively the purity of gold alloys. The experimental system was composed of a radioactive source ${ }^{152} \mathrm{Eu}$ (31 MBq) emitting many $\boldsymbol{\gamma}$-ray energies and a high-purity germanium detector having a high-energy resolution. The TEGAT technique suggested that the purity of gold alloys of 1-5 mm thickness can be determined with a precision of $>99 \%$ for an allowance range condition of two karats. By the DEGAT technique, however, the purity of gold alloys of 3-5 mm thickness could be determined with 95-99\% precision under the same conditions as above. For the DEGAT technique, it was assumed in this study that the mass attenuation coefficient of silver was equal to that of copper. The $\gamma$ ray transmission techniques can be effectively applied for the measurements of the purities of thick gold alloys which are otherwise impossible to determine by $\mathrm{X}$-ray fluorescence analysis.

\section{CATALYSIS}

\subsection{Gold-Based Catalyst for Exhaust Gas Purification}

L.A. Petrov of the Laman Consultancy Limited, Bulgaria has filed a patent (WO Patent 9851401 A1 19981119) claiming gold catalysts for exhaust gas treatment. The invention relates to a gold catalyst for the oxidation of carbon monoxide and hydrocarbons, reduction of nitrous oxides and degradation of ozone, for use in the protection of the environment. The active component of the catalyst consists of a complex of gold and reducible transition metal oxide. The concentration of gold in the catalyst is from 0.1 to $2.5 \%$ and the total concentration of the metals in the active component of the catalyst is from 0.1 to $10 \%$ of the total mass. The support consists of ceria and titania. The surface area of the catalyst is between 80 and $400 \mathrm{~m}^{2} / \mathrm{g}$ and the catalyst working temperature is from 0 to $400^{\circ} \mathrm{C}$.

\subsection{Characterization of Alumina-Supported Gold using Temperature-Programmed Reduction}

C.-K. Chang, Y.J. Chen and C.T. Yeh of the Department of Chemistry, National Tsinghua University, Hsinchu, Taiwan, P.R. China (Appl. Catal, A, 1998, 174, 13-23) prepared supported $\mathrm{Au} / \mathrm{Al}_{2} \mathrm{O}_{3}$ samples of different gold loadings by the deposition-precipitation of $\mathrm{AuCl}_{3}$ onto alumina. These samples were characterized using atomic emission (ICP), $\mathrm{x}$-ray diffraction (XRD), transmission electron microscopy (TEM) and temperature-programmed reduction (TPR). According to TEM and $x$-ray diffraction observations, the size of gold crystallites dispersed on samples freshly prepared at $300 \mathrm{~K}$ was ca $8 \mathrm{~nm}$. TPR characterization of oxidized samples gave three kinds of reductive peaks at temperatures of ca 180, 300 and $1000 \mathrm{~K}$. These peaks were attributed to reductions of gold oxides $\left(\mathrm{Au}_{\mathrm{s}} \mathrm{O}_{\mathrm{x}}\right)$ and gold chloride $\left(\mathrm{Au}_{\mathrm{s}} \mathrm{Cl}_{\mathrm{y}}\right)$ on the surface of supported gold crystallites, and of gold ions incorporated into the subsurface of the alumina support, respectively. The stoichiometry $\mathrm{x}$ of $\mathrm{Au}_{\mathrm{s}} \mathrm{O}_{\mathrm{x}}$ increased with the temperature of oxidative treatment and attained a peak value of 2.0 at around $600 \mathrm{~K}$. Using this adsorption stoichiometry, variations in the dispersions of gold crystallites on alumina were determined. Through 
dispersion measurements, supported nano-crystallites of gold were found to sinter at $673 \mathrm{~K}$ in both oxidative and reductive environments.

\subsection{Investigation of Small Size-Controlled Gold Particles for Low Temperature CO-Oxidation by Electron Microscopy and Related Investigations}

J.-D. Grunwaldt, O.S. Becket, A. Baiker and B. Calderon; ed. A. Hector and M. Jose Yacaman, of the Laboratory of Technical Chemistry, Swiss Federal Institute of Technology, ETH-Zentrum, Zurich, Switzerland (Electron Microsc. 1998, Proc. Int. Congr., $\left.14^{\text {th }}, 1998,2,431-432\right)$ describe the preparation of gold catalysts on different supports using gold colloids, which offers the advantage that the final gold size is established before the deposition. TEM and UV-visible spectrophotometry are used to establish the gold particle size in solution and on the support (See also J.-D. Grunwaldt, C. Keiner, C. Wögerbauer and A. Baiker, J. Catal., 1999, 181, 223).

In another recent paper J.-D. Grunwaldt and A. Baiker ( J. Phys. Chem. B, 1999, 102, 1002) the chemisorption properties of differently prepared model gold/titania interfaces have been compared with the aim of gaining a better understanding of the synergistic interplay between the constituents in gold/titania catalysts used in low temperature carbon monoxide oxidation. As a result a mechanistic model was proposed which is based on oxygen adsorption on vacancy sites of titania and $\mathrm{CO}$ adsorption on gold.

\subsection{Supported Gold Catalysis Derived from the Interaction of a Gold-Phosphine Complex with as-Precipitated Titanium Hydroxide and Titanium Oxide}

Supported gold catalysts derived from the interaction of $\mathrm{Au}\left(\mathrm{PPh}_{3}\right)\left(\mathrm{NO}_{3}\right)$ (1) with conventional titania and as-precipitated titanium hydroxide $\mathrm{Ti}(\mathrm{OH})_{4}{ }^{*}\left(^{*}=\right.$ as- $^{-}$ precipitated) have been characterized using XRD, XPS, EXAFS, and ${ }^{31} \mathrm{P}$ CP/MAS-NMR (Y. Yuan, K. Asakura, A.P. Kozlova, H. Wan, K. Tsai and Y. Iwasawa, Department of Chemistry, The University of Tokyo; Catal. Today, 1998, 44, 333-342). The gold complex 1 was supported on $\mathrm{TiO}_{2}$ and $\mathrm{Ti}(\mathrm{OH})_{4}{ }^{*}$ without loss of gold-phosphorus bonding at room temperature. The gold complex 1 on $\mathrm{TiO}_{2}$ was readily and completely decomposed to form metallic gold particles by calcination at $473 \mathrm{~K}$, whereas only a small part of the complex 1 on $\mathrm{Ti}(\mathrm{OH})_{4}{ }^{*}$ was transformed to metallic gold particles. By calcination of $1 / \mathrm{Ti}(\mathrm{OH})_{4}{ }^{*}$ at $573 \mathrm{~K}$ the formation of both metallic gold particles and crystalline titanium oxides became notable as detected by XRD, XPS and ${ }^{31} \mathrm{P}$ CP/MAS-NMR. The mean diameter of gold particles in $1 / \mathrm{Ti}(\mathrm{OH})_{4}{ }^{*}$ calcined at $673 \mathrm{~K}$ was less than $30 \AA$ as estimated from $\mathrm{Au}(2 \quad 0 \quad 0)$ diffraction, which was about one-tenth of that for the corresponding $1 / \mathrm{TiO}_{2}$. Thus the asprecipitated titanium hydroxide $\mathrm{Ti}(\mathrm{OH})_{4}{ }^{*}$ was able to stabilize the gold complex 1 to lead to the simultaneous decomposition of gold complex and $\mathrm{Ti}(\mathrm{OH})_{4}{ }^{*}$. The catalyst $1 / \mathrm{Ti}(\mathrm{OH})_{4}{ }^{*}$ calcined at $673 \mathrm{~K}$ afforded remarkably high catalytic activity for lowtemperature $\mathrm{CO}$ oxidation at $273-373 \mathrm{~K}$ as compared to the catalyst $1 / \mathrm{TiO}_{2}$.

\section{CHEMISTRY}

\subsection{Preparation of and Crystal Structure for $\mathrm{K}_{3} \mathrm{Au}_{5} \mathrm{~Pb}$}

Silver-coloured, brittle single crystals of $\mathrm{K}_{3} \mathrm{Au}_{5} \mathrm{~Pb}$ were synthesized by reaction of $\mathrm{KN}_{3}$ with gold sponge and lead powder at $920 \mathrm{~K}$ (U. Zachwieja and J. Wlodarski, Fachbereich Chemie, Universität Dortmund, Germany; $Z$. Anorg. Allg. Chem.,1998, 624, 1569-1572). The Au-Pb partial structure consists of layers of gold tetrahedra and lead zigzag chains with $\mathrm{Pb}$ atoms which are connected to a framework structure via $\mathrm{Au}-\mathrm{Pb}$ contacts. The $\mathrm{K}$ atoms occupy channels within the $\mathrm{Au}-\mathrm{Pb}$ partial structure.

\subsection{Synthesis, Structures, and Properties of New Quaternary Gold Chalcogenides, $\mathrm{K}_{2} \mathrm{Au}_{2} \mathrm{Ge}_{2} \mathrm{~S}_{6}, \mathrm{~K}_{2} \mathrm{Au}_{2} \mathrm{Sn}_{2} \mathrm{Se}_{6}$, and $\mathrm{Cs}_{2} \mathrm{Au}_{2} \mathrm{SnS}_{4}$}

$\mathrm{S}$. Löken and W. Tremel of the Institut Anorganische Chemie Analytische Chemie, Universität Mainz, Germany (Z. Anorg. Allg. Chem.,1998, 624, 15881594) describe the synthesis of the new compounds $\mathrm{K}_{2} \mathrm{Au}_{2} \mathrm{Ge}_{2} \mathrm{~S}_{6}$ (I), $\mathrm{K}_{2} \mathrm{Au}_{2} \mathrm{Sn}_{2} \mathrm{Se}_{6}$ (II), and $\mathrm{Cs}_{2} \mathrm{Au}_{2} \mathrm{SnS}_{4}$ (III) via direct reaction of the elements with a molten polyalkali thiogermanate(stannate) flux at 650,550 , and $400^{\circ} \mathrm{C}$. Their crystal structures were determined by single crystal $\mathrm{x}$-ray diffraction techniques. The structures of I, II, and III consist of infinite anionic chains linked by $\mathrm{Au}^{+}$ions and charge balancing $\mathrm{K}^{+} / \mathrm{Cs}^{+}$ ions situated between the chains. All compounds were investigated with DTA, FT-IR, and solid-state UV/VIS diffuse reflectance spectroscopy.

\subsection{Ca $\mathrm{C}_{2} \mathrm{AuN}$ : a Nitride Containing Infinite Zigzag Gold Chains}

A polycrystalline ternary calcium auride nitride $\mathrm{Ca}_{2} \mathrm{AuN}$ was prepared by reaction of $\mathrm{Ca}_{3} \mathrm{~N}_{2}$ powder 
with gold foil under high-pressure of dry nitrogen (P.F. Henry and M.T. Weller, Department of Chemistry, University of Southampton, UK, Angew. Chem., Int. Ed., 1998, 37, 2855-2857). The compound was structurally characterized from Rietveld refinement of powder neutron and XRD data. The most distinctive feature is the presence of infinite, planar zigzag gold chains parallel to the crystallographic c axis.

\section{COATINGS, FILMS, MEMBRANES AND WIRES}

\subsection{Chemisorption and Chemical Reaction Effects on the Resistivity of Ultrathin Gold Films at the Liquid-Solid Interface}

Y. Zhang, R.H. Terrill and P.W. Bohn of the Department of Chemistry, Beckman Institute and Materials Research Laboratory, University of Illinois at Urbana-Champaign, USA (Anal. Chem.,1999, 71, 119-125) have reported that ultrathin gold films, with thicknesses between the onset of conduction (diameter ca $5 \mathrm{~nm}$ ) and the electron mean free path (diameter $c a$ $80 \mathrm{~nm}$ ), display surface-sensitive resistivities, which have been exploited to follow the adsorption and desorption of molecular monolayers at the metalsolution interface with high precision. For nominal gold film thicknesses (ca $40 \mathrm{~nm}$ ), strongly chemisorbed thiolate monolayers increase the resistivity of the thin gold films by ca $4 \%$, but weakly adsorbed species, such as pyridine or phenolate at open circuit, induce no observable change in the gold film resistance.

Surface plasmon resonance measurements, performed simultaneously with resistivity measurements, indicate that changes in resistivity vary monotonically with coverage with three distinct regions: a low-coverage region of heightened adsorbate mobility, an intermediate-coverage region with generally linear behaviour, and a chain lengthdependent saturation region at high coverages. Resistivity measurements were capable of reproducibly following the chemical state of the gold surface through a complex set of redox manipulations, demonstrating the versatility of this simple measurement.

\subsection{Evidence of Pure Diffusion Process During Growth of Gold Films}

The surface roughness of evaporated gold films has been studied by scanning tunnelling microscopy and analysed by a Fourier transform method (A.I. Oliva, J.L. Sacedon, E. Anguiano, M. Aguilar, J.A. Aznarez and J.A. Mendez,
Instituto Ciencia Materiales (CSIC), Campus Universidad Autonoma, Madrid, Spain; Surf. Sci., 1998, 417, L1139-L1142). The power spectrum presents a clear dependence in the high-frequency region and exponential time behaviour of the total interface width of the films. The values obtained for the two exponents, in the range of $300-18000 \AA$ thickness studied are in agreement with the theoretical predictions for a pure diffusion-controlled process but not with a re-evaporation process. The power spectrum for high frequencies shows a correlation for different film thicknesses describing scaling without anomalous behaviour.

\section{COLLOIDS AND NANOPARTICLES}

\section{$5.1 C_{60}$-Mediated Aggregation of Gold Nanoparticles}

Aggregation of gold particles in presence of fullerene has been reported by M. Brust, C.J. Kiely, D. Bethell and D.J. Schiffrin from the Department of Chemistry, The University of Liverpool, UK (J.Am. Chem. Soc., 1998, 120, 12367-12368), based on TEM and HRTEM spectroscopy. The spectroscopic images suggest that fullerene $\left(\mathrm{C}_{60}\right)$ monolayers were adsorbed on ca $5 \mathrm{~nm}$ diameter gold nanoparticles stabilized by adsorption of bromide and quaternary ammonium ions in toluene solution. The formation of selfassembled monolayers of fullerene on immobilized gold particles has been observed and examined by SERS. The origin of the binding interaction between gold particles and fullerene molecules is unclear, but the observation of an ESR spectrum similar to that observed on electrochemical reduction of $C_{60}$ suggests an involvement of an electron-transfer process.

\subsection{Formation and Stability of Gold Sol Produced from Chloroauric Acid using Citric Acid}

The formation of gold sol from chloroauric acid by addition of citric acid has been studied by Y. Mori, Y. Murao and Y. Miyake of the Department of Chemical Engineering and Materials Science, Doshisha University, Kyotanabe, Japan (World Congr. Part. Technol., 1998, 3, 1831-1841; available on disk - Publisher: Institution of Chemical Engineers, Rugby, UK) using spectrophotometry. Absorption band of the gold sol appeared in the wavelength range from 500 to $600 \mathrm{~nm}$. The gold sol formation rate increased as the $\mathrm{pH}$ decreased. The induction period, which was related to the period of gold cluster formation, increased with the concentration of chloroauric acid. Particle size, measured 
by small angle $\mathrm{x}$-ray scattering and dynamic light scattering, decreased with increases in the concentrations of hydrogen citrate ion and chloroauric acid and was controlled by those concentrations. The stability of colloidal gold depended on the $\mathrm{pH}$ which was related to the concentration of citrate ion. An addition of sodium hydroxide after the formation of colloidal gold would be a suitable way to make a stable gold sol.

\subsection{Preparation and Characterization of Dendrimer-Gold Colloid Nanocomposites}

M.E. Garcia, L.A. Baker and R.M. Crooks of the Department of Chemistry, Texas A\&M University, College Station, Texas (Anal. Chem., 1999, 71, 256-258) have prepared gold colloids in the 2-3 nm size regime by in situ reduction of $\mathrm{HAuCl}_{4}$ in the presence of poly(amidoamine) dendrimers. The dendrimers encapsulate the colloids, imparting stability to the aqueous colloidal solutions. The nanocomposite materials can be isolated by precipitation. The dendrimer generation used in the synthesis controls the size of the resultant colloids: lower-generation dendrimers give rise to larger colloids. The materials were characterized by IR and UV-visible spectroscopy and transmission electron microscopy.

\subsection{Enormous Hyper-Rayleigh Scattering from Nanocrystalline Gold Particle Suspensions}

The recent emergence of advanced technological applications for colloidal gold suspensions and related particle assemblies and interfaces has created a demand for new chemical and physical techniques with which to characterize them (F.W. Vance, B.I. Lemon and J.T. Hupp, Department of Chemistry and Materials Research Center, Northwestern University, Evanston, USA; J. Phys. Chem. B, 1998, 102, 1009110093). For macroscopic samples/interfaces, coherent second harmonic generation (SHG) proved itself a useful characterization tool due, at least in part, to metal-based plasmon enhancement. In an effort to defeat or bypass the size restrictions inherent in SHG, the authors used a related incoherent methodology, hyper-Rayleigh scattering (HRS), to interrogate aqueous colloidal suspensions of $13 \mathrm{~nm}$ diameter gold particles.

The nanoscale particles proved to be remarkably efficient scatterers; when evaluated in terms of the first hyperpolarizability $(\beta)$, HRS signals from the gold particles substantially surpass those observable from the best available molecular chromophores. The present experiments indicate that $\beta$ is highly sensitive to colloid aggregation and imply that HRS is an effective tool for the characterization of symmetry-reducing perturbations of nanoscale interfaces.

\subsection{The Shape Transition of Gold Nanorods}

S.-S. Chang, C.-W. Shih, C.-D. Chen, W.-C. Lai and C.R.R. Wang of the Department of Chemistry, National Chung Cheng University, Chia-Yi, Taiwan report a revised synthetic procedure based on an electrochemical method for preparing an aqueous solution containing suspended gold nanorods (Langmuir, 1999, 15, 701-709). The mean aspect ratios of the gold nanorods can be experimentally adjusted between 1 and 7 . The evolution of the longitudinal surface plasmon bands shows an eminently sensitive dependence on the aspect ratios of the nanorods. Their dependence is accordingly described by classical-electrostatic-model predictions. The shape transition of the nanorod particles was studied by varying some key influencing factors such as the wavelength, the laser fluence, and matrix effects. The nanorods were exposed to laser lines at 532 and $1064 \mathrm{~nm}$, frequencies which correspond closely to the short- and long-axis plasmon resonances, respectively. A photon-induced shape transition process was in evidence, and the corresponding rod-to-sphere conversion contributed by a photoannealing process was observed in both cases.

Meanwhile, the authors observed a new type of $\phi-$ shaped gold nanostructure in the case of $1064 \mathrm{~nm}$ irradiation, which possibly represents the early stage of the shape transition and indicates that the starting location of the atomic-scale restructuring is at the centroid of the gold nanorod. The results of laser fluence-dependence measurements state that an efficient shape transition occurs via a multiphoton process. The authors also demonstrate the fabrication of the gold nanorod silica nanostructures for preliminary studies of the matrix effects. As a result of the higher rigidity of the thin silica coating layer, the associated shape transition requires higher energy and proceeds less efficiently as compared with the cases for the micelle-stabilized gold nanorods.

\section{ELECTROCHEMISTRY}

\subsection{Environmental-Compatible Gilding. A New Bath System for Electroless Gold Deposition on Electronics Components}

A new cyanide-free bath for electroless gold deposition has been developed (J. Müller, R. Schmidt, H. Griese, 
M. Hannemann and K.H. Zuber, Fraunhofer-Institut Zuverlaessigkeit Mikrointegration, Berlin; Metalloberfläche, 1998, 52, 873-875). This not only fulfils the technical requirements but also uses materials which are not toxic, are environmentally friendly, and are biologically degradable. The properties of the new bath are compared with conventional baths.

\subsection{Hydrogen Evolution Reaction on Gold Single-Crystal Electrodes in Acid Solutions}

J. Perez, E.R. Gonzalez and H.M. Villullas, Instituto de Quimica de Sao Carlos, Brazil (J. Phys. Chem. B, 1998, 102, 10931-10935) have studied the hydrogen evolution reaction on the low index planes of singlecrystal gold electrodes in $\mathrm{HClO}_{4}$ solutions with the aim of ascertaining the extent of the sensitivity of the reaction rate to the surface crystallographic structure. The use of the hanging meniscus rotating disk technique allowed the carrying out of all measurements under rotation, thus avoiding blocking effects produced by hydrogen bubbles and the accumulation of dissolved hydrogen in the interfacial region during potential excursions into relatively high overpotentials. In contrast with previously published results showing only slight variations in activity with gold crystal face, a pronounced structural sensitivity is reported for the first time.

The catalytic activity has the following sequence $\mathrm{Au}(111)>\mathrm{Au}(100)>\mathrm{Au}(110)$.

\section{ELECTRONICS}

\subsection{The Effect of Thin Film Structure and Properties on Gold Ball Bonding}

The gold ball bonding process is widely used for making interconnections between integrated circuit chips and package lead frames, yet the relations between the wire/substrate materials properties and the bond formation processes are not yet well understood. While the creation of a metallurgical bond at the interface between the wire and substrate is required, the deformation of the wire and substrate also play an important role in bond formation. Bonding to thin film substrates is of particular interest since thin films often exhibit mechanical behaviour distinctly different from bulk materials. A systematic study was conducted by J.E. Krzanowski, E. Razon and A.F. Hamiel of the Mechanical Engineering Department, University of New Hampshire, Durham, USA (J. Electron. Mater, 1998, 27, 1211-1215) in order to understand the effects of the structure and properties of aluminium thin films on the quality of gold ball bonds.

Aluminium thin films were fabricated with systematic variations in hardness, roughness, thickness, and composition. Gold wires were ball bonded to these substrates, and the bondability and bond shear strengths were assessed. Metallographic sections of several of these specimens were made and examined in the scanning electron microscope. The film thickness has the most dominant effect on the bondability and bond strength; films that were $0.5 \mu \mathrm{m}$ thick often exhibited low strength or poor bondability. Very hard films also gave poor results. Ultimately, these results can be used to predict the wire bond reliability expected from various types of thin film metalization.

\subsection{The Usage of Focused Ion Beam Induced Deposition of Gold Film in IC Device Modification and Repair}

R. Lee and M. Cecere, of Schlumberger ATE, San Jose, California (ISTFA '97, Proc. Int. Symp. Test. Failure Anal., 1997, 23, 121-123 (Publisher: ASM International, Materials Park, Ohio) consider that as semiconductor processes become more advanced, Focused Ion Beam (FIB) surgery techniques need to develop to match the new challenges. One area for performance enhancement is in the deposition of lower resistivity conducting materials. At the present time, the two most commonly used materials for conductive deposition are platinum and tungsten. The main issue concerning these two materials is the relatively high resistivity of the deposited material; the deposited film can have 100 to 200 times the resistivity of the pure material. FIB induced deposition of gold films is commonly used in the repair of masks. In this application the resistivity of the deposited sample is of little importance.

An examination of $\mathrm{C}_{7} \mathrm{H}_{10} \mathrm{AuF}_{3} \mathrm{O}_{2}$ (tfac) as a precursor gas shows that its resistivity when deposited with a FIB is $4-5$ times lower than that of platinum or tungsten. The deposition rate is also significantly quicker. No electrical-migration and dendrite formation was noticed after the gold deposition was subjected to continuous current flow. However, the thermal stability of the pre-cursor gas is low and will need further refinement.

\subsection{Fabrication of Colloidal Gold Micropatterns using Photolithographed Self-Assembled Monolayers as Templates}

A paper by J.F. Liu, L.-G. Zhang, N. Gu, J.Y. Ren, Y.-P. Wu, Z.H. Lu, P.S. Mao and D.Y. Chen (National 
Laboratory of Molecular and Biomolecular Electronics, Southeast University, Nanjing, P.R. China; Thin Solid Films, 1998, 327-329, 176-179) provides a new method for the patterned metalization of self-assembled monolayers. Colloidal gold micropatterns were formed on photolithographed self-assembled monolayers (SAMs) of (3-mercaptopropyl)-trimethoxysilane (MTS) on silicacoated silicon substrates. As confirmed by SEM, the colloidal gold closely replicated the mask features. Atomic force microscopy (AFM) showed that the colloidal gold particles on the unirradiated monolayers of (3-mercaptopropyl)-trimethoxysilane were connected and an almost continuous monolayer of gold nanoparticles was formed. X-ray photoelectron microscopy (XPM) revealed that the $-\mathrm{SH}$ terminal groups of the MTS monolayer in the exposed region were oxidized and the self-assembly of colloidal gold onto the unexposed region were through Au-S covalent bonding. The present approach may be applicable to assembly of microelectronic circuits and microbiosensors.

\subsection{Formation of Silicon-Gold Eutectic Bond using Localized Heating Method}

L. Lin, Y.T. Cheng and K. Najafi, Department of Mechanical Engineering and Applied Mechanics, University of Michigan, USA (Jpn. J. Appl. Phys., Part 2, 1998, 37(11B), L1412-L1414) have described a new bonding technique by using localized heating to supply the bonding energy. Heating is achieved by applying a d.c. current through micromachined heaters made of gold which serves as both the heating and bonding material. At the interface between silicon and gold, the formation of the eutectic bond takes place in about 5 minutes. Assembly of two substrates in microfabrication processes can be achieved by using this method. In this paper, the following significant results are reported: (1) gold diffuses into silicon to form a strong eutectic bond by means of localized heating; (2) the bonding strength reaches the fracture toughness of the bulk silicon; (3) this bonding technique greatly simplifies the device fabrication and assembly processes.

\section{MATERIALS SCIENCE}

\subsection{Optical Properties of Gold in Acetate Glasses}

The colours of gold ruby glasses are variable, depending in part on the sizes of the gold particles dispersed in the colloidal state throughout the silicate glass. N.R. Wilk and H.D. Schreiber, Department of
Chemistry, Virginia Military Institute, Lexington, USA (J. Non-Cryst. Solids, 1998, 239, 192-196) have demonstrated that the incorporation of gold into leadlithium acetate glasses and sodium-potassium-calcium acetate glasses gives colours varying from pink to purple, as the colloidal dispersion is affected by the structure of the acetate system. The structural dependence of the colour of gold in these acetate systems is unlike that for the dependence of the colour of $\mathrm{Co}^{2+}$ in the same acetates.

\subsection{Paraconductivity in $\mathrm{YBa}_{2} \mathrm{Cu}_{3} \mathrm{O}_{7}$ and $\mathrm{YBa}_{2} \mathrm{Cu}_{3} \mathrm{O}_{7}$ Thin Film Composites with Cerium Oxide and Gold}

E.J. Cukauskas and L.H. Allen of the Electronics Science and Technology Division, Naval Research Laboratory, Washington, DC, USA (J. Appl. Phys., $1998,84,6187-6194)$ have studied and analysed the paraconductivity for thin films of $\mathrm{YBa}_{2} \mathrm{Cu}_{3} \mathrm{O}_{7}$ (YBCO) and composites of $\mathrm{YBCO}$ with $\mathrm{CeO}_{2}$ and gold using the Aslamazov and Larkin theory. Plain YBCO thin films sputter deposited on $\mathrm{MgO}, \mathrm{SrTiO}_{3}$, and $\mathrm{Y}$ stabilized zirconia typically showed no dimensional crossover, were described by three dimensional (3D) fluctuation conductivity, and had a critical slope just above the critical region in agreement with the prediction of theory. With few exceptions, the $\mathrm{CeO}_{2}$ and gold composite films showed a dimensional crossover and a reduced critical exponent. The change in dimensionality was typically from three-dimensional to two dimensional (2D) fluctuation conductivity These composite films all had reduced values for the critical exponent which the authors attribute to an enlarged or extended critical region. This is in agreement with the critical current response of these composites to small magnetic fields. In addition the composites showed a two step transition in the paraconductivity and a minimum below the critical region. The subsequent fall-off to the zero resistance state was characterized by two different slopes. Lastly, the paraconductivity was related to film degradation resulting from photoresist processing and time storage. Dimensionality changes were observed due to this degradation.

\section{METALLURGY}

\subsection{Lattice Strains in Gold and Rhenium under Non-Hydrostatic Compression}

Lattice strains have been measured as a function of the angle from the diamond cell stress axis in gold and rhenium specimens at pressures of 15-37 GPa by T.S. 
Duffy, G. Shen, D.L. Heinz, Y. Ma, R.J. Hemley, H.K. Mao and A.K. Singh of the Department of Geosciences, Princeton University, NJ, USA (Mater. Res. Soc. Symp. Proc., 1998, 499 (High-Pressure Materials Research), 145-150). Experiments were conducted by using $\mathrm{x}$-ray transparent gaskets made from beryllium. The differential stresses supported by gold and rhenium have been characterized to $37 \mathrm{GPa}$. Proper choice of the diffraction geometry allows recovery of a quasi-hydrostatic compression curve under these highly non-hydrostatic conditions. X-ray elastic moduli have also been determined, and while agreement with previous data was achieved for gold, there was a large discrepancy between the present results and extrapolated ultrasonic data for rhenium.

\subsection{UHV Electron Microscope and Simultaneous STM Observation of Gold Stepped Surfaces}

H. Ohnishi, Y. Kondo and K. Takayanagi of the Takayanagi Particle Surface Project, Japan Science and Technology Corporation, Tokyo (Surf. Sci., 1998, 415, L1061-L1064) have used a miniaturized scanning tunneling microscope (STM) in a UHV electron microscope to simultaneously observe specimen surfaces in transmission electron microscope images and STM images. Gold (111) stepped surfaces were observed with a gold STM tip with a spherical apex curvature of $16 \mathrm{~nm}$. The tip positions were measured from the electron microscope images recorded on a VTR during the STM observation. It was found that the tip position changed coincidentally with the STM profile and that the shortest distance between the stepped (111) surface of the island and the spherical tip apex was kept constant.

\subsection{Thermal Stability of Nanocrystalline Gold Studied by X-ray Diffraction Method}

The grain size of nanocrystalline gold specimens prepared by a gas deposition method has been measured using x-ray diffraction (T. Inami, S. Okuda, $\mathrm{H}$. Maeta and H. Ohtsuka, Faculty of Engineering, Ibaraki University, Japan; Mater. Trans., JIM, 1998, 39, 1029-1032). The grain size of as-prepared specimens, estimated by the Warren-Averbach method, was in the range $7-11 \mathrm{~nm}$, and no apparent dependence on the evaporation temperature (1700-1850 K) and helium gas pressure $(0.085-0.186 \mathrm{MPa})$ in the evaporation chamber was found. The thermal stability of these specimens was high. When annealed for $1 \mathrm{~h}$, the grain size remained unchanged at $770 \mathrm{~K}$, grew by a factor of ca 2 at $1070 \mathrm{~K}$, and displayed rapid growth at higher temperatures.

\section{REFINING}

\subsection{A Study of Various Methods for Cyanide Destruction by Oxidation of Gold Leach Residues from a South African Gold Mine, and Subsequent Flotation of Pyrite}

This paper by A.M. Ribeiro Botelho de Sousa of the Instituto Geologico e Mineiro Laboratory, Mamede de Infesta, Portugal (Estud., Notas Trab. Inst. Geol. Min., $1997,39,91-98)$ is the summary of a study carried out at Mintek (Council for Mineral Technology), in South Africa, envisaging the economic viability of an alkaline pyrite flotation circuit, at a gold mine, in order to replace the existing traditional acidic circuit, which had become barely profitable when in the mid-eighties, the uranium prices decreased to such an extent that its recovery was no longer advantageous. The work consisted of testing various methods for cyanide removal by oxidation of the gold leach residues, the float plant feed, followed by a comparative study of alkaline vs acidic flotation of the gold-bearing pyrite. A replacement of the acidic flotation circuit for an alkaline xanthate flotation after cyanide oxidation following the Inco $\mathrm{SO}_{2} / \mathrm{Air}$ process at $\mathrm{pH} 8$, was recommended, as the latter was shown to be not only feasible but also cheaper to carry out.

\subsection{Gold Hydrometallurgy: Theory and Practice}

M.A. Reuter of the Faculty of Applied Earth Sciences Delft, Delft University of Technolgy, The Netherlands (Schriftenr. GDMB, 1998, 82 (Fortschritte in der Hydrometallurgie), 233-268) has written this review, with 34 references on the leaching of gold from freemilling, complex, and refractory ores and the subsequent recovery of gold from the solution (filtration/precipitation, carbon-in-pulp (CIP), carbonin-leach (CIL), and resin-in-pulp (RIP) methods). Topics discussed in gold leaching include reaction equations, cyanide leaching chemistry, factors influencing the cyanide leaching of free milling gold ores, leaching of complex ores, refractory ores, leaching kinetics, and leaching models. 